\title{
Shortcuts to adiabaticity: Fast-forward approach
}

\author{
E. Torrontegui, ${ }^{1}$ S. Martínez-Garaot, ${ }^{1}$ A. Ruschhaupt, ${ }^{2}$ and J. G. Muga ${ }^{1,3}$ \\ ${ }^{1}$ Departamento de Química Física, Universidad del País Vasco - Euskal Herriko Unibertsitatea, Apdo. 644, Bilbao, Spain \\ ${ }^{2}$ Institut für Theoretische Physik, Leibniz Universität Hannover, Appelstraße 2, 30167 Hannover, Germany \\ ${ }^{3}$ Department of Physics, Shanghai University, 200444 Shanghai, People's Republic of China
}

(Received 16 April 2012; published 3 July 2012)

\begin{abstract}
The "fast-forward"approach by Masuda and Nakamura generates driving potentials to accelerate slow quantum adiabatic dynamics. First we present a streamlined version of the formalism that produces the main results in a few steps. Then we show the connection between this approach and inverse engineering based on Lewis-Riesenfeld invariants. We identify in this manner applications in which the engineered potential does not depend on the initial state. Finally we discuss more general applications exemplified by wave splitting processes.
\end{abstract}

DOI: 10.1103/PhysRevA.86.013601

PACS number(s): 03.75.Kk, 42.50.Dv, 37.10.Gh

\section{INTRODUCTION}

Motivated by the practical need to accelerate quantum adiabatic processes in different contexts (transport [1-5], expansions [6,7], population inversion and control [8-13], cooling cycles $[6,14,15]$, and wave-function splitting [16-19]), and by related fundamental questions (about the quantum limits to the speed of processes, the viability of adiabatic computing [20], or the third principle of thermodynamics [14,21]), a flurry of theoretical and experimental activity has been triggered by the proposal of several approaches to design "shortcuts to adiabaticity". Among other approaches let us mention (i) a transitionless tracking algorithm or "counterdiabatic" approach that adds to the original Hamiltonian extra terms to cancel transitions in the adiabatic or superadiabatic bases [8-13]; (ii) inverse engineering of the external driving [3,4,6,21-26] based on Lewis-Riesenfeld invariants [27], which has been applied in several expansion experiments [25,26]; (iii) optimal control (OC) methods $[5,7,14,16]$, sometimes combined with other methods to enhance their performance [4,5,7]; (iv) the fast-forward (FF) approach advocated by Masuda and Nakamura [19,28]; (v) parallel adiabatic passage [29-32].

The multiplicity of approaches is quite useful because they may complement each other: either in the same application, as demonstrated, e.g., with OC and invariant-based methods, or because of their different domains. Clarifying the features, overlaps, and relations among these approaches is important to apply the ones which are best suited for specific systems and objectives [33], or to develop new ones. In this paper we shall establish in particular the connection between the fast-forward and the invariant-based methods.

Based on some earlier results [34], the fast-forward formalism for adiabatic dynamics and several application examples were worked out in Refs. [19,28] by Masuda and Nakamura for the Gross-Pitaevskii or the corresponding Schrödinger equations. The objective of the method is to accelerate a "standard" system subjected to a slow variation of external parameters. The time is then rescaled by a "magnification factor", and an ansatz wave function is defined by the standard function multiplied by a phase factor that, in general, depends on position and time. Inserting the ansatz into the dynamical equation provides in principle the form of the necessary fast-forward driving potential and the equation to be satisfied by the phase. This procedure uses a tricky cancellation between the divergence due to the infinitely large magnification factor and the infinitesimal slowness due to adiabaticity.

As a consequence of the different steps and functions introduced the resulting procedure is somewhat involved, which hinders a broader application. In Sec. II we provide a streamlined construction of local and real fast-forward potentials, and Sec. III delves into a more detailed connection between this streamlined version and the original formulation of the FF formalism. Section IV relates the fast-forward approach to the inverse method for dynamical invariants which are quadratic in momentum. Section V discusses applications beyond this domain, in particular wave-function splitting, which is an important operation for matter wave interferometry [16-18,35]. Finally Sec. VI discusses the results and open questions.

\section{A SIMPLE INVERSE METHOD}

Our starting point is the three-dimensional (3D) timedependent Gross-Pitaevskii (GP) equation,

$$
i \hbar \frac{\partial|\psi(t)\rangle}{\partial t}=H(t)|\psi(t)\rangle,
$$

where the Hamiltonian $H$ is the sum of the kinetic energy $T$, the external potential $V(t)$, and the mean field potential $G(t)$. We are assuming an external local potential, where "local" means here $\left\langle\mathbf{x}|V(t)| \mathbf{x}^{\prime}\right\rangle=V(\mathbf{x}, t) \delta\left(\mathbf{x}-\mathbf{x}^{\prime}\right)$. The kinetic and mean field terms in the coordinate representation have the usual forms,

$$
\begin{aligned}
\langle\mathbf{x}|T| \psi(t)\rangle & =\frac{-\hbar^{2}}{2 m} \nabla^{2} \psi(\mathbf{x}, t), \\
\langle\mathbf{x}|G(t)| \psi(t)\rangle & =g|\psi(\mathbf{x}, t)|^{2} \psi(\mathbf{x}, t) .
\end{aligned}
$$

The GP equation (1) is used to describe a Bose-Einstein condensate within the mean field approximation and it takes into account the atom-atom interaction through $g$, the atomatom coupling constant. In the case of vanishing coupling constant $g=0$ the GP equation simplifies to the Schrödinger equation.

By solving Eq. (1) in coordinate space, $V(\mathbf{x}, t)$ may be written as

$$
V(\mathbf{x}, t)=\frac{i \hbar\left\langle\mathbf{x} \mid \partial_{t} \psi(t)\right\rangle-\langle\mathbf{x}|T| \psi(t)\rangle-\langle\mathbf{x}|G(t)| \psi(t)\rangle}{\langle\mathbf{x} \mid \psi(t)\rangle},
$$


with $\langle\mathbf{x} \mid \psi(t)\rangle=\psi(\mathbf{x}, t)$. By introducing into Eq. (2) the ansatz

$$
\langle\mathbf{x} \mid \psi(t)\rangle=r(\mathbf{x}, t) e^{i \phi(\mathbf{x}, t)}, \quad r(\mathbf{x}, t), \phi(\mathbf{x}, t) \in \mathbb{R},
$$

we get

$$
\begin{aligned}
V(\mathbf{x}, t)= & i \hbar \frac{\dot{r}}{r}-\hbar \dot{\phi}+\frac{\hbar^{2}}{2 m}\left(\frac{2 i \nabla \phi \cdot \nabla r}{r}+i \nabla^{2} \phi\right. \\
& \left.-(\nabla \phi)^{2}+\frac{\nabla^{2} r}{r}\right)-g r^{2},
\end{aligned}
$$

where the dot means time derivative. The real and imaginary parts are

$$
\begin{gathered}
\operatorname{Re}[V(\mathbf{x}, t)]=-\hbar \dot{\phi}+\frac{\hbar^{2}}{2 m}\left(\frac{\nabla^{2} r}{r}-(\nabla \phi)^{2}\right)-g r^{2}, \\
\operatorname{Im}[V(\mathbf{x}, t)]=\hbar \frac{\dot{r}}{r}+\frac{\hbar^{2}}{2 m}\left(\frac{2 \nabla \phi \cdot \nabla r}{r}+\nabla^{2} \phi\right) .
\end{gathered}
$$

Our purpose now is to design a local and real potential such that an initial eigenstate of the initial Hamiltonian, typically the ground state but it could be otherwise, evolves in a time $t_{f}$ into the corresponding eigenstate of the final Hamiltonian (a different goal will be discussed in the final section). We assume that the full Hamiltonian and the corresponding eigenstates are known at the boundary times.

By construction the potential of Eq. (4) is local. If we impose $\operatorname{Im}[V(\mathbf{x}, t)]=0$, i.e.,

$$
\frac{\dot{r}}{r}+\frac{\hbar}{2 m}\left(\frac{2 \nabla \phi \cdot \nabla r}{r}+\nabla^{2} \phi\right)=0,
$$

then we get from Eq. (5) a local and real potential.

In the inversion protocol we design $r(\mathbf{x}, t)$ first, then solve for $\phi$ in Eq. (7), and finally get the potential $V$ from Eq. (5). If, at the boundary times, $\dot{r}=0$ is imposed, Eq. (7) has solutions $\phi(\mathbf{x}, t)$ fulfilling that $\phi(\mathbf{x}, t)$ is independent of $\mathbf{x}$ at $t=0$ and $t=t_{f}$. Using this in Eq. (5) at $t=0$, and multiplying by $e^{i \phi(0)}$, we get

$$
\begin{aligned}
& {\left[-\frac{\hbar^{2}}{2 m} \nabla^{2}+V(\mathbf{x}, 0)+g|\psi(\mathbf{x}, 0)|^{2}\right] \psi(\mathbf{x}, 0)} \\
& =-\hbar \dot{\phi}(0) \psi(\mathbf{x}, 0) .
\end{aligned}
$$

The initial state is thus an eigenstate of the stationary GP equation at $t=0$, and $-\hbar \dot{\phi}(0)=E(0)$ is the energy of the eigenstate $\psi(\mathbf{x}, 0)$. Note that the above solution of $\phi$ (with $\dot{r}=0$ at boundary times) admits the addition of an arbitrary function that depends only on time and modifies the zero of energy. A similar result is found at $t_{f}$.

\section{CONNECTION WITH THE FAST-FORWARD APPROACH}

The aim of the fast-forward approach $[19,34]$ is to accelerate a standard system subjected to a slow, adiabatic variation of external parameters by canceling the divergence due to an infinitely large magnification factor with the infinitesimal slowness due to adiabaticity. In this manner a fast-forward potential is constructed which leads to the speeded-up evolution of the system.

Let us now look at this approach in more detail and connect it with the above results. The notation is made close but not necessarily in full agreement with $[19,34]$. Let us define an external parameter that depends on time, or on some scaled time function, according to

$$
R(\Lambda(t))=\epsilon \Lambda(t)=: \mathcal{R}(t) .
$$

Here $\mathcal{R}(t)$ and $R(\Lambda)$ are in general different functions of their arguments, $\epsilon$ is a small positive constant, and the scaling function $\Lambda(t)$ is given in terms of a magnification factor $\alpha$,

$$
\Lambda(t)=\int_{0}^{t} d t^{\prime} \alpha\left(t^{\prime}\right)
$$

$\alpha(t)$ is positive for $0 \leqslant t \leqslant t_{f}$ and zero at the boundaries $t=0$ and $t=t_{f}$. Note that $\dot{\mathcal{R}}=\epsilon \alpha$ and $\ddot{\mathcal{R}}=\epsilon \dot{\alpha}$. We rewrite the modulus and the phase in Eq. (3) as

$$
\begin{gathered}
r(\mathbf{x}, t)=\tilde{r}(\mathbf{x}, \mathcal{R}(t)), \\
\phi(\mathbf{x}, t)=-\frac{1}{\hbar} \int_{0}^{t} d t^{\prime} \mathcal{E}\left(\mathcal{R}\left(t^{\prime}\right)\right)+\epsilon \alpha(t) \theta(\mathbf{x}, \mathcal{R}(t)),
\end{gathered}
$$

where again we have distinguished the functions according to their different arguments, in particular $E(t)=\mathcal{E}(\mathcal{R}(t))$. If we also demand $\dot{\alpha}=0$ at the boundaries to fulfill $\dot{r}=$ 0 , then $\dot{\phi}(\mathbf{x}, 0)=-\mathcal{E}(\mathcal{R}(0)) / \hbar$ and $\dot{\phi}\left(\mathbf{x}, t_{f}\right)=-\mathcal{E}\left(\mathcal{R}\left(t_{f}\right)\right) / \hbar$. Substituting Eq. (12) in Eq. (7), $\theta$ has to satisfy

$$
\begin{aligned}
0= & \tilde{r}(\mathbf{x}, \mathcal{R}(t)) \nabla^{2} \theta(\mathbf{x}, \mathcal{R}(t))+2 \nabla \tilde{r}(\mathbf{x}, \mathcal{R}(t)) \cdot \nabla \theta(\mathbf{x}, \mathcal{R}(t)) \\
& +\frac{2 m}{\hbar} \frac{\partial \tilde{r}}{\partial \mathcal{R}}(\mathbf{x}, \mathcal{R}(t))
\end{aligned}
$$

and from Eq. (5), the "fast-forward" potential is given by

$$
\begin{aligned}
V(\mathbf{x}, t)= & V_{0}(\mathbf{x}, \mathcal{R}(t))-\hbar \epsilon \dot{\alpha}(t) \theta(\mathbf{x}, \mathcal{R}(t)) \\
& -\hbar \epsilon^{2} \alpha^{2}(t) \frac{d \theta}{d \mathcal{R}}(\mathbf{x}, \mathcal{R}(t)) \\
& -\frac{\hbar^{2}}{2 m} \epsilon^{2} \alpha^{2}(t)[\nabla \theta(\mathbf{x}, \mathcal{R}(t))]^{2},
\end{aligned}
$$

where the "standard potential" $V_{0}=V_{0}(\mathbf{x}, \mathcal{R})$ is defined by the stationary GP equation,

$$
\left[-\frac{\hbar^{2}}{2 m} \nabla^{2}+V_{0}(\mathbf{x}, \mathcal{R})+g \tilde{r}^{2}(\mathbf{x}, \mathcal{R})\right] \tilde{r}(\mathbf{x}, \mathcal{R})=\mathcal{E}(\mathcal{R}) \tilde{r}(\mathbf{x}, \mathcal{R}) .
$$

At the boundary times, but in general only there, $V(\mathbf{x}, t)=$ $V_{0}(\mathbf{x}, \mathcal{R}(t))$.

Equation (14) for the driving potential coincides with Eq. (2.28) in Ref. [19] for real eigenfunctions, whereas Eq. (13) for the phase function $\theta$ corresponds to Eq. (2.18) in Ref. [19].

The present formal framework may be used in the following way: (i) starting from a given standard potential $V_{0}(\mathbf{x}, \mathcal{R})$, $\tilde{r}(\mathbf{x}, \mathcal{R})$, and $\mathcal{E}(\mathcal{R})$ would follow from Eq. (15). Alternatively, it is also possible to impose $\tilde{r}(\mathbf{x}, \mathcal{R})$ first and then calculate $V_{0}$. (ii) An auxiliary function $\mathcal{R}(t)$ is imposed. (iii) $\theta$ has to be determined from Eq. (13). (iv) The fast-forward potential can be calculated from Eq. (14).

To arrive at this recipe in Refs. [19,34] preliminary steps are the definition of a standard state, a virtually fast-forwarded state, and a regularized state with their corresponding equations. The route followed in Sec. II to the driving potential is in comparison quite direct. This is so because we made no explicit use of a slow reference adiabatic process, although it 
might be deduced from the fast designed dynamics if required. The key simplification is to start with the ansatz in Eq. (3) and derive the two basic equations for phase and potential from it by imposing locality and reality of the driving potential. Since the phase $\phi$ that solves Eq. (7) depends in general on the particular $r(\mathbf{x}, t)$, the potential calculated through Eqs. (5) or (14) gives in principle a state-dependent potential. However, in some special circumstances, as we shall see below, the fast-forward potential becomes state independent.

\section{CONNECTION WITH INVARIANT'S BASED INVERSE ENGINEERING APPROACH}

In this section we shall relate the previous results for the linear $(g=0)$ Schrödinger equation to the engineering approach based on quadratic-in-momentum invariants. The nonlinear GP equation could also be treated, as in Refs. [4,22], but it does not allow in general for the state-independent potential forms that we shall describe for $g=0$.

\section{A. Lewis-Leach potentials}

In a direct (rather than inverse) approach, the potential $V(\mathbf{x}, t)$ is considered to be known, and the wave function at any time $t$ can be deduced from the Lewis-Riesenfeld theory of invariants [27]. Suppose that the potential $V(\mathbf{x}, t)$ has the structure of the most general "Lewis-Leach" potential that admits a quadratic-in-momentum invariant [36],

$$
V(\mathbf{x}, t)=-\mathbf{F}(t) \cdot \mathbf{x}+\frac{1}{2} m \omega^{2}(t)|\mathbf{x}|^{2}+\frac{1}{\rho^{2}} U(\boldsymbol{\sigma})+h(t),
$$

where $\omega(t), \mathbf{F}(t)$, and $h(t)$ are arbitrary functions of time and $U(\sigma)$ is an arbitrary function of its argument $\sigma=\sigma(t)=(\mathbf{x}-$ $\boldsymbol{\alpha}) / \rho$. The time dependent functions $\rho=\rho(t)$ and $\boldsymbol{\alpha}=\boldsymbol{\alpha}(t)$ must satisfy the auxiliary equations,

$$
\begin{aligned}
\frac{\omega_{0}^{2}}{\rho^{3}} & =\ddot{\rho}+\omega^{2}(t) \rho, \\
\frac{\mathbf{F}(t)}{m} & =\ddot{\boldsymbol{\alpha}}+\omega^{2}(t) \boldsymbol{\alpha},
\end{aligned}
$$

with $\omega_{0}$ an arbitrary constant. The associated dynamical invariant, up to a constant factor, is given by

$$
I=\frac{1}{2 m}|\rho(\mathbf{p}-m \dot{\boldsymbol{\alpha}})-m \dot{\rho}(\mathbf{x}-\boldsymbol{\alpha})|^{2}+\frac{1}{2} m \omega_{0}^{2}|\boldsymbol{\sigma}|^{2}+U(\boldsymbol{\sigma}),
$$

with $\mathbf{p}=-i \hbar \nabla$. It satisfies $d I / d t=\partial I(t) / \partial t-\frac{i}{\hbar}[I(t)$, $H(t)]=0$, so its expectation values are constant for any wave function $\psi(t)$ that evolves with $H$.

For the potential in Eq. (16), the general solution of the time-dependent Schrödinger equation, Eq. (1), can be expanded as a linear combination with constant coefficients $c_{n}$ and orthonormal eigenvectors $\psi_{n}$ of $I$ [27],

$$
\begin{gathered}
\psi(\mathbf{x}, t)=\sum_{n} c_{n} e^{i \alpha_{n}} \psi_{n}(\mathbf{x}, t), \\
I \psi_{n}(\mathbf{x}, t)=\lambda_{n} \psi_{n}(\mathbf{x}, t),
\end{gathered}
$$

where $\lambda_{n}$ are the time independent eigenvalues of $I$. The phases $\alpha_{n}$ satisfy $\hbar \frac{d \alpha_{n}}{d t}=\left\langle\psi_{n}\left|i \hbar \frac{\partial}{\partial t}-H\right| \psi_{n}\right\rangle[3,4,27]$,

$$
\alpha_{n}=-\frac{i}{\hbar} \int_{0}^{t} d t^{\prime}\left(\frac{\lambda_{n}}{\rho^{2}}+\frac{m\left[|\dot{\boldsymbol{\alpha}} \rho-\boldsymbol{\alpha} \dot{\rho}|^{2}-\omega_{0}^{2}|\boldsymbol{\alpha}|^{2} / \rho^{2}\right]}{2 \rho^{2}}+h\right) .
$$

Performing now the unitary transformation $[3,4]$,

$$
\psi_{n}(\mathbf{x}, t)=e^{\frac{i m}{\hbar}\left[\dot{\rho}|\mathbf{x}|^{2} / 2 \rho+(\dot{\alpha} \rho-\boldsymbol{\alpha} \dot{\rho}) \cdot \mathbf{x} / \rho\right]} \frac{1}{\rho^{3 / 2}} \chi_{n}(\boldsymbol{\sigma}),
$$

the state $\psi_{n}$ is easily obtained from the solution $\chi_{n}(\sigma)$ (normalized in $\sigma$ space) of the auxiliary stationary Schrödinger equation,

$$
\left[-\frac{\hbar^{2}}{2 m} \nabla_{\sigma}^{2}+\frac{1}{2} m \omega_{0}^{2}|\sigma|^{2}+U(\sigma)\right] \chi_{n}(\boldsymbol{\sigma})=\lambda_{n} \chi_{n}(\boldsymbol{\sigma}) .
$$

In the direct approach we assume that $U(\boldsymbol{\sigma}), \omega(t)$, and $\mathbf{F}(t)$ are known. Solving Eqs. (17) and (18) we get $\rho(t)$ and $\alpha(t)$ from them. Thus Eq. (24) can be solved to get $\lambda_{n}$ and $\chi_{n}(\sigma)$. Finally, combining Eqs. (23) and (24), the mode $e^{i \alpha_{n}} \psi_{n}$ can be calculated at any time.

\section{B. Inverse engineering approach}

In the inverse approach based on quadratic-in-momentum invariants, the Hamiltonian is assumed to have the form given in Eq. (16), at all times and in particular at initial and final instants. As $U$ is given the stationary Eq. (24) may be solved. Then the functions $\rho$ and $\alpha$ are designed so that $[H(t), I(t)]=$ 0 for $t=0$ and $t=t_{f}$. Thus the Hamiltonian and the invariant have common eigenvectors at these boundary times [3-7,23,24]. Typically the initial state $\psi(0)$ is the ground state of $H(0)$ which is also an eigenstate of $I(0)$, and this state evolves according to Eq. (20), as an eigenvector of the invariant.

To relate the above to the simple inverse method of Sec. II we consider the single mode wave function $\psi_{n}$ of Eq. (23) and identify

$$
r_{n}(\mathbf{x}, t)=\chi_{n}(\boldsymbol{\sigma}) / \rho^{3 / 2}(t)
$$

The subscript $n$ underlines the dependence with the $n$th mode considered. Note that $\rho$ and $\boldsymbol{\alpha}$ are chosen at this point. We may get the phase $\phi_{n}$ from Eq. (7). It can be checked by direct substitution that

$$
\begin{aligned}
\phi_{n}= & \frac{m}{\hbar}\left[\dot{\rho}|\mathbf{x}|^{2} / 2 \rho+(\dot{\boldsymbol{\alpha}} \rho-\boldsymbol{\alpha} \dot{\rho}) \cdot \mathbf{x} / \rho\right] \\
& -\frac{1}{\hbar} \int_{0}^{t} d t^{\prime} \frac{\lambda_{n}}{\rho^{2}}+\mathcal{F}(t),
\end{aligned}
$$

where

$$
\mathcal{F}(t)=-\frac{1}{\hbar} \int_{0}^{t} d t^{\prime}\left(\frac{m\left[|\dot{\boldsymbol{\alpha}} \rho-\boldsymbol{\alpha} \dot{\rho}|^{2}-\omega_{0}^{2}|\boldsymbol{\alpha}|^{2} / \rho^{2}\right]}{2 \rho^{2}}+h\right)
$$

is a solution of this equation. Once $r_{n}$ and the phase $\phi_{n}$ are known, Eq. (5) gives the potential $V_{n}(\mathbf{x}, t)$. A different arbitrary function of time $\mathcal{F}(t)$ in $\phi_{n}(\mathbf{x}, t)$ would produce a shift of the zero of energy in the resulting potential $V_{n}(\mathbf{x}, t)$. We get, using 
Eq. (24),

$$
\begin{aligned}
V(\boldsymbol{x}, t)= & -m\left(\ddot{\boldsymbol{\alpha}}+\boldsymbol{\alpha} \frac{\omega_{0}^{2}+\rho^{3} \ddot{\rho}}{\rho^{4}}\right) \boldsymbol{x} \\
& +\frac{m}{2}\left(\frac{\omega_{0}^{2}+\rho^{3} \ddot{\rho}}{\rho^{4}}\right)|\boldsymbol{x}|^{2}+\frac{1}{\rho^{2}} U(\boldsymbol{\sigma})+h .
\end{aligned}
$$

Taking Eqs. (17) and (18) into account, this potential agrees with the potential in Eq. (16). It is by construction local and real. Moreover, it is independent of the $n$th state considered so that linear combinations of the modes at $t=0$ end up at $t_{f}$ unexcited, preserving the initial populations.

We have seen so far that the simple inverse method, a streamlined FF approach, may be used to calculate from a given wave evolution directly the required potentials consistent with this evolution. The inverse method is a general procedure to achieve this potential reconstruction for the GP Eq. (1). In general, the resulting potentials are - by construction state-dependent. By contrast, the invariant-based inverse engineering for quadratic-in-momentum invariants is restricted to the Lewis-Leach potentials of Eq. (16), but an advantage is that the resulting potentials are state-independent. In other words, final excitations will be avoided for any initial state. As we have shown above, the invariant-based approach can be formulated as a special case of the simple inverse method.

So, the inverse engineering approach and the fast-forward approach are connected via the simple inverse method. Therefore, the external parameter $\mathcal{R}$ introduced in Sec. III must be related to $\boldsymbol{\alpha}$ and $\rho$, as illustrated in the following section.

\section{Example: Harmonic expansion}

Now we discuss an example of a 3D harmonic expansion produced with the inverse engineering approach based on invariants and with the fast-forward technique to illustrate the links between the two methods.

Invariants based approach. Suppose that the expansion is governed by the Hamiltonian

$$
H(t)=\frac{\mathbf{p}^{2}}{2 m}+\frac{1}{2} m \omega^{2}(t)|\boldsymbol{x}|^{2},
$$

where $\omega(t)$ is unknown, but at the boundary times $\omega(0)=\omega_{0}$ and $\omega\left(t_{f}\right)=\omega_{f}$. This potential is a particular case of Eq. (16) with $\mathbf{F}(t)=U(\boldsymbol{\sigma})=h(t)=0$. Equation (18) is trivially fulfilled if $\boldsymbol{\alpha}(t)=\ddot{\boldsymbol{\alpha}}(t)=0$ and consequently $\dot{\boldsymbol{\alpha}}(t)=0 . \rho(t)$ has to satisfy the Ermakov equation, Eq. (17). The inverse engineering consists on imposing conditions on $\rho$ and its derivatives,

$$
\begin{array}{cl}
\rho(0)=1, & \dot{\rho}(0)=0, \quad \ddot{\rho}(0)=0, \\
\rho\left(t_{f}\right)=\gamma, \quad \dot{\rho}\left(t_{f}\right)=0, \quad \ddot{\rho}\left(t_{f}\right)=0,
\end{array}
$$

where $\gamma=\left(\omega_{0} / \omega_{f}\right)^{1 / 2}$, to guarantee the commutation between $H(t)$ and $I(t)$ at $t=0$ and $t_{f}$, and then getting $\omega(t)$ from Eq. (17).

Fast-forward approach. The starting point for the fastforward approach could be the $n$th eigenstate of a harmonic trap (see also Ref. [19]) with angular frequency $\mathcal{R}=\omega$,

$$
\begin{aligned}
\chi_{n}(\mathbf{x}, \mathcal{R})= & \frac{\beta^{3 / 2} e^{-\frac{\beta^{2} \mid x x^{2}}{2}}}{\pi^{3 / 4} \sqrt{2^{n_{x}+n_{y}+n_{z}} n_{x} ! n_{y} ! n_{z} !}} H_{n_{x}}(\beta x) H_{n_{y}}(\beta y) \\
& \times H_{n_{z}}(\beta z),
\end{aligned}
$$

where $\beta=\sqrt{m \mathcal{R} / \hbar}$. This state plays the role of $\tilde{r}$. The corresponding potential $V_{0}$ is clearly

$$
V_{0}(x, \mathcal{R})=\frac{m}{2} \mathcal{R}^{2} \mathbf{x}^{2},
$$

and

$$
\mathcal{E}_{n}=\hbar \omega\left(n_{x}+n_{y}+n_{z}+\frac{3}{2}\right) .
$$

The first step is to solve Eq. (13) and we get as a solution

$$
\theta(\mathbf{x}, \mathcal{R})=-\frac{m|\mathbf{x}|^{2}}{4 \hbar \mathcal{R}} .
$$

Connection. The connection between the auxiliary variable $\mathcal{R}$ in the fast-forward approach and the auxiliary variable $\rho(t)$ in the inverse engineering approach is in this example explicitly given by

$$
\mathcal{R}(t)=\frac{\omega_{0}}{\rho(t)^{2}}
$$

[see Eqs. (25) and (30)]. The boundary conditions for $\rho(t)$ in Eq. (29) become

$$
\begin{gathered}
\mathcal{R}(0)=\omega_{0}, \quad \dot{\mathcal{R}}(0)=0, \quad \ddot{\mathcal{R}}(0)=0, \\
\mathcal{R}\left(t_{f}\right)=\omega_{f}, \quad \dot{\mathcal{R}}\left(t_{f}\right)=0, \quad \ddot{\mathcal{R}}\left(t_{f}\right)=0 .
\end{gathered}
$$

It also follows that $\epsilon \alpha(t)=\dot{\mathcal{R}}(t)=-2 \omega_{0} \dot{\rho}(t) / \rho(t)^{3}$. The auxiliary functions $\rho(t)$ and $\mathcal{R}(t)$ can be chosen in some way fulfilling the boundary conditions.

The corresponding potential in the inverse engineering formalism is constructed by first solving the Ermakov equation to get $\omega^{2}(t)$. Then one has

$$
V=\frac{1}{2} m \omega^{2}(t)|\mathbf{x}|^{2}=\frac{1}{2} m\left(\frac{\omega_{0}^{2}}{\rho(t)^{4}}-\frac{\ddot{\rho}}{\rho}\right)|\mathbf{x}|^{2},
$$

whereas the fast-forward potential is given, according to Eq. (14), by

$$
\begin{aligned}
V= & \frac{m|\mathbf{x}|^{2}}{2}\left(\mathcal{R}^{2}+\hbar \epsilon \dot{\alpha} \frac{m}{4 \hbar \mathcal{R}}-\hbar \epsilon^{2} \alpha^{2} \frac{m}{4 \hbar \mathcal{R}^{2}}\right. \\
& \left.-\frac{\hbar^{2}}{2 m} \epsilon^{2} \alpha^{2} \frac{m^{2}}{4 \hbar^{2} \mathcal{R}^{2}}\right) \\
= & \frac{m|\mathbf{x}|^{2}}{2}\left(\frac{\omega_{0}^{2}}{\rho(t)^{4}}-\frac{\ddot{\rho}(t)}{\rho(t)}\right),
\end{aligned}
$$

which agrees with Eq. (36).

\section{BEYOND LEWIS-LEACH POTENTIALS: WAVE-FUNCTION SPLITTING PROCESSES}

The transitionless condition for the inverse engineering method based on invariants relies on the commutativity $[H(t), I(t)]=0$ at times $t=0$ and $t_{f}$, which guarantees common eigenvectors for $H$ and $I$ at these boundary times. According to Eq. (23) the structure of the density of the $n$th 


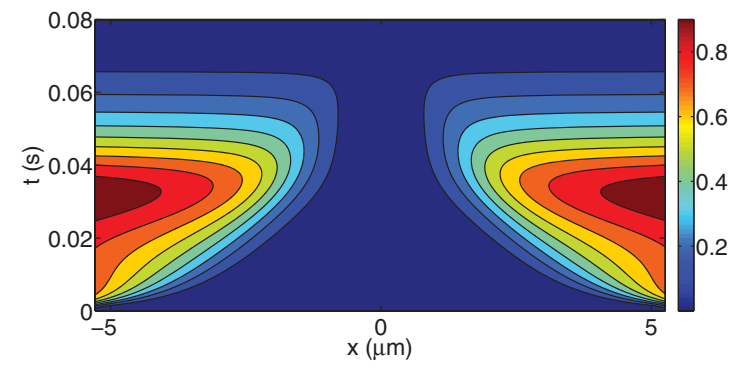

FIG. 1. (Color online) Phase $\phi(x, t)$ calculated from Eq. (6). Parameters: $m=1.44 \times 10^{-25} \mathrm{~kg}, a=3 \mu \mathrm{m}, \omega / 2 \pi=125 \mathrm{~Hz}$ and $t_{f}=80 \mathrm{~ms}$.

mode of the invariant at initial and final times for quadratic-in-p invariants is

$$
\frac{1}{\rho^{3}(0)}\left|\chi_{n}\left(\frac{\mathbf{x}-\boldsymbol{\alpha}(0)}{\rho(0)}\right)\right|^{2} \rightarrow \frac{1}{\rho^{3}\left(t_{f}\right)}\left|\chi_{n}\left(\frac{\mathbf{x}-\boldsymbol{\alpha}\left(t_{f}\right)}{\rho\left(t_{f}\right)}\right)\right|^{2}
$$

The final density is a translation and/or scaling of the initial one. This means that for processes in which the initial and final eigenstates of the Hamiltonian do not behave according to Eq. (37), the commutativity of $H$ and $I$ at the boundary times cannot be achieved. This restriction is due to the use of quadratic-in-p invariants, not to the invariants-based method. Studying and applying more general invariants is still an open question.

As an example in which Eq. (37) does not hold for the final densities, let us consider the splitting of an initial state from a single to a double well potential. For simplicity we take the 1D linear Schrödinger equation governed by the Hamiltonian,

$$
H(t)=\frac{p^{2}}{2 m}+\frac{1}{2} m \omega^{2}(t) x^{2}+\eta(t) x^{4} .
$$

For the initial single trap we consider $\omega^{2}(0)=\omega_{0}^{2}$ and $\eta(0)=\eta_{0}>0$. The final double well is characterized by a repulsive harmonic part with $\omega^{2}\left(t_{f}\right)=-\omega_{f}^{2}$ and $\eta\left(t_{f}\right)=$ $\eta_{f}>0$. Comparing terms with Eq. (16), $F=h=\alpha=0$ and consequently $\dot{\alpha}=\ddot{\alpha}=0, U(\sigma)=\eta(t) \rho^{2} x^{4}$, and the particular structure of $U(\sigma)$ sets $\eta(t)=\kappa / \rho^{6}$, where $\rho$ satisfies the Ermakov equation, Eq. (17), and $\kappa$ is an arbitrary constant. The associated invariant is

$$
I(t)=\frac{1}{2 m}(\rho p-m \dot{\rho} x)^{2}+\frac{1}{2} m \omega_{0}^{2} \frac{x^{2}}{\rho^{2}}+\kappa \frac{x^{4}}{\rho^{4}} .
$$

Imposing $\rho(0)=1, \dot{\rho}(0)=0, \ddot{\rho}(0)=0$, and identifying $\kappa=$ $\eta_{0}$, then $H$ and $I$ commute at $t=0$. At $t_{f},\left[H\left(t_{f}\right), I\left(t_{f}\right)\right]=0$ for $\quad \rho\left(t_{f}\right)=\left(i \omega_{0} / \omega_{f}\right)^{1 / 2}, \quad \dot{\rho}\left(t_{f}\right)=\ddot{\rho}\left(t_{f}\right)=0, \quad$ and $\eta_{f}=-i \eta_{0} \omega_{f}^{3} / \omega_{0}^{3}$. However, $\rho$ must be a positive real function if initially so, and moreover the final potential that we get is complex.

A way out is to use the simple inverse fast-forward method for specific initial and final states without restricting the potential form; see also [19]. Consider, for example, the 1D splitting of the initial state $r(x, 0)=e^{-\beta^{2} x^{2} / 2}(\beta=\sqrt{m \omega / \hbar})$ into the final form $r\left(x, t_{f}\right)=e^{-\beta^{2}(x-a)^{2} / 2}+e^{-\beta^{2}(x+a)^{2} / 2}$. In between we apply the interpolation

$$
r(x, t)=z(t)\left\{[1-\mathcal{R}(t)] r(x, 0)+\mathcal{R}(t) r\left(x, t_{f}\right)\right\},
$$

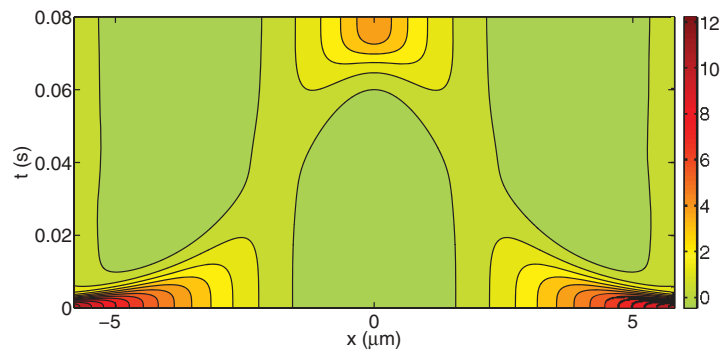

FIG. 2. (Color online) Fast-forward potential $V(x, t)$ in units of $\hbar \omega$ for a final time $t_{f}=80 \mathrm{~ms}$. The rest of parameters are the same as in Fig. 1. Note the coexistence of three wells, e.g., around $40 \mathrm{~ms}$, before the final two wells are established.

where $\mathcal{R}(t)$ is some smooth, monotonously increasing function from 0 to 1 and $z(t)$ is a normalization function. We also impose that $\dot{\mathcal{R}}=0$ to ensure $\dot{r}=0$ at the boundary times $t=0$ and $t_{f}$. In the numerical examples the function $\mathcal{R}(t)$ is chosen as a polynomial of degree 7 to make zero the second and third derivatives at the boundaries. Once we have established the form of $r(x, t)$, we solve Eq. (6), $\operatorname{Im}[V(x, t)]=0$, to get the phase $\phi$ with the initial conditions $\phi(0, t)=\phi^{\prime}(0, t)=0$ that fix the zero-energy point (the prime means spatial derivative). Then the resulting phase is introduced into Eq. (5) to get the potential $V(x, t)$. In Fig. 1 the phase $\phi(x, t)$ is plotted for a nonadiabatic process with $t_{f}=80 \mathrm{~ms}$. The corresponding fastforward potential is plotted in Fig. 2.

$V(x, t)$ in Fig. 2 could be realized with high resolution time-varying optical potentials "painted" by a tightly focused rapidly moving laser beam [37], or by means of spatial light modulators [38]. A simpler approximate approach would involve the combination of three Gaussian beams. In principle the time $t_{f}$ can be reduced to produce the splitting in a shorter time. For example, in Fig. $3 t_{f}=10 \mathrm{~ms}$ and a more complicated potential is needed.

\section{DISCUSSION}

We have first distilled from the somewhat imposing set of equations of the fast-forward (FF) formalism as originally presented a streamlined version that may aid to apply it more easily. Our second aim has been to relate it to other inverse engineering methods. In a previous publication, the inverse-engineering method based on invariants was related to the transitionless tracking algorithm [8-11], and their potential equivalence was demonstrated [33]. Similarly, we have established in this paper the connection between the

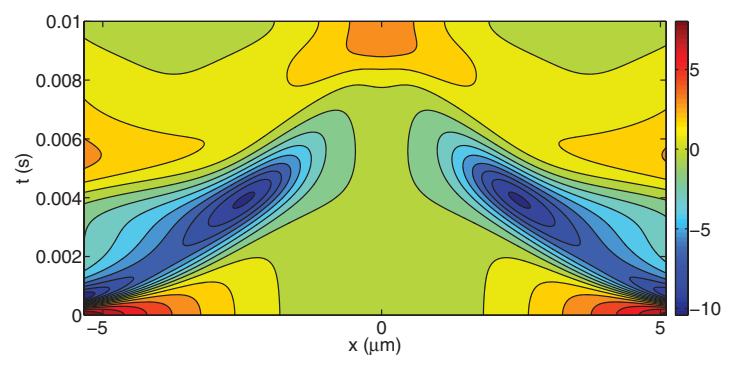

FIG. 3. (Color online) Fast-forward potential $V(x, t)$ in units of $\hbar \omega$ for a final time $t_{f}=10 \mathrm{~ms}$. The rest of parameters are the same as in Fig. 1. 
fast-forward method and the invariant-based method for quadratic-in-momentum invariants. These relations do not imply the full identity of the methods but their overlap and equivalence in a common domain. They are still useful heuristically as separate approaches since they are formulated in rather different terms [28,33]. Moreover, they facilitate extensions beyond their common domain, as exemplified by the wave-splitting processes discussed in the previous section.

Further extensions are left for separate analysis: for example, the possibility to transfer an excited state into the ground state or vice versa, or combining the fast-forward approach with optimal control theory without including the final fidelity in the cost function as in Refs. [16-18]. (This would be possible because the fidelity is guaranteed to be one by construction.) It will also be interesting for future work to consider complex potentials, either as solutions to the shortcut dynamics, as in the quantum brachistochrone [39], or as an effective description of the system dynamics to be accelerated [23]. Finally, while we have concentrated on wave equations and functions in coordinate space, inverse techniques are also applicable to two-level or more general discrete-level systems. Garanin, for example, performed inverse engineering for the Landau-Zener model [40]. The transitionless-tracking algorythm was in fact originally applied in for these systems [8-11], and its potential equivalence with the invariant-based approach also holds in this context [33]. Designing a specific wave function in the spirit of the streamlined FF method to engineer the Hamiltonian matrix implies the dynamical invariant of the corresponding density operator. Details of these connections will appear elsewhere.

\section{ACKNOWLEDGMENTS}

We are grateful to S. Masuda and K. Nakamura for discussing their method; also to G. Labeyrie for comments on experimental techniques. We acknowledge funding by Projects No. GIU07/40 and No. FIS2009-12773-C02-01, and the UPV/EHU under program UFI 11/55. E.T. acknowledges financial support from the Basque Government (Grant No. BFI08.151).
[1] A. Couvert, T. Kawalec, G. Reinaudi, and D. Guéry-Odelin, Eur. Phys. Lett. 83, 13001 (2008).

[2] M. Murphy, L. Jiang, N. Khaneja, and T. Calarco, Phys. Rev. A 79, 020301(R) (2009).

[3] E. Torrontegui, S. Ibáñez, X. Chen, A. Ruschhaupt, D. GuéryOdelin, and J. G. Muga, Phys. Rev. A 83, 013415 (2011).

[4] E. Torrontegui, X. Chen, M. Modugno, S. Schmidt, A. Ruschhaupt, and J. G. Muga, New J. Phys. 14, 013031 (2012).

[5] X. Chen, E. Torrontegui, D. Stefanatos, J.-S. Li, and J. G. Muga, Phys. Rev. A 84, 043415 (2011).

[6] X. Chen, A. Ruschhaupt, S. Schmidt, A. del Campo, D. GuéryOdelin and J. G. Muga, Phys. Rev. Lett. 104, 063002 (2010).

[7] D. Stefanatos, J. Ruths, and J.-S. Li, Phys. Rev. A 82, 063422 (2010).

[8] M. Demirplak and S. A. Rice, J. Chem. Phys. A 107, 9937 (2003).

[9] M. Demirplak and S. A. Rice, J. Phys. Chem. B 109, 6838 (2005).

[10] M. Demirplak and S. A. Rice, J. Chem. Phys. 129, 154111 (2008).

[11] M. V. Berry, J. Phys. A 42, 365303 (2009).

[12] X. Chen, I. Lizuain, A. Ruschhaupt, D. Guéry-Odelin, and J. G. Muga, Phys. Rev. Lett. 105, 123003 (2010).

[13] M. G. Bason, M. Viteau, N. Malossi, P. Huillery, E. Arimondo, D. Ciampini, R. Fazio, V. Giovannetti, R. Mannella, and O. Morsch, Nature Phys. 8, 147 (2012).

[14] P. Salamon, K. H. Hoffmann, Y. Rezek, and R. Kosloff, Phys. Chem. Chem. Phys. 11, 1027 (2009).

[15] K. H. Hoffmann, P. Salamon, Y. Rezek, and R. Kosloff, Europhys. Lett. 96, 60015 (2011).

[16] U. Hohenester, P. K. Rekdal, A. Borzi, and J. Schmiedmayer, Phys. Rev. A 75, 023602 (2007).

[17] J. Grond, J. Schmiedmayer, and U. Hohenester, Phys. Rev. A 79, 021603 (2009).

[18] J. Grond, G. von Winckel, J. Schmiedmayer, and U. Hohenester, Phys. Rev. A 80, 053625 (2009).

[19] S. Masuda and K. Nakamura, Proc. R. Soc. A 466, 1135 (2010).
[20] H. T. Quan and W. H. Zurek, New J. Phys. 12, 093025 (2010).

[21] X. Chen and J. G. Muga, Phys. Rev. A 82, 053403 (2010).

[22] J. G. Muga, X. Chen, A. Ruschhaupt, and D. Guéry-Odelin, J. Phys. B 42, 241001 (2009).

[23] S. Ibáñez, S. Martínez-Garaot, X. Chen, E. Torrontegui, and J. G. Muga, Phys. Rev. A 84, 023415 (2011).

[24] E. Torrontegui, X. Chen, M. Modugno, A. Ruschhaupt, D. Guéry-Odelin, and J. G. Muga, Phys. Rev. A 85, 033605 (2012).

[25] J. F. Schaff, X. L. Song, P. Vignolo, and G. Labeyrie, Phys. Rev. A 82, 033430 (2010).

[26] J. F. Schaff, X. L. Song, P. Capuzzi, P. Vignolo, and G. Labeyrie, Eur. Phys. Lett. 93, 23001 (2011).

[27] H. R. Lewis and W. B. Riesenfeld, J. Math. Phys. 10, 1458 (1969).

[28] S. Masuda and K. Nakamura, Phys. Rev. A 84, 043434 (2011).

[29] S. Guérin, S. Thomas, and H. R. Jauslin, Phys. Rev. A 65, 023409 (2002).

[30] G. S. Vasilev, A. Kuhn, and N. V. Vitanov, Phys. Rev. A 80, 013417 (2009).

[31] G. Dridi, S. Guérin, V. Hakobyan, H. R. Jauslin, and H. Eleuch, Phys. Rev. A 80, 043408 (2009).

[32] S. Guérin, V. Hakobyan, and H. R. Jauslin, Phys. Rev. A 84, 013423 (2011).

[33] X. Chen, E. Torrontegui, and J. G. Muga, Phys. Rev. A 83, 062116 (2011).

[34] S. Masuda and K. Nakamura, Phys. Rev. A 78, 062108 (2008).

[35] L. Pezze, A. Smerzi, G. P. Berman, A. R. Bishop, and L. A. Collins, New J. Phys. 7, 85 (2005).

[36] H. R. Lewis and P. G. Leach, J. Math. Phys. 23, 2371 (1982).

[37] K. Henderson, C. Ryu, C. MacCormick, and M. G. Boshier, New J. Phys. 11, 043030 (2009).

[38] V. Boyer, R. M. Godun, G. Smirne, D. Cassettari, C. M. Chandrashekar, A. B. Deb, Z. J. Laczik and, C. J. Foot, Phys. Rev. A 73, 031402(R) (2006).

[39] C. M. Bender, D. C. Brody, H. F. Jones, and B. K. Meister, Phys. Rev. Lett. 98, 040403 (2007).

[40] D. A. Garanin and R. Schilling, Europhys. Lett. 59, 7 (2002). 